\title{
The optical-near-infrared colour of the host galaxies of BL Lacertae objects ${ }^{\star}$
}

\author{
J. K. Kotilainen ${ }^{1}$ and R. Falomo ${ }^{2}$ \\ 1 Tuorla Observatory, University of Turku, Väisäläntie 20, 21500 Piikkiö, Finland \\ e-mail: jarkot@utu.fi \\ 2 INAF - Osservatorio Astronomico di Padova, Vicolo dell'Osservatorio 5, 35122 Padova, Italy \\ e-mail: falomo@pd.astro.it
}

Received 26 February 2004 / Accepted 24 May 2004

\begin{abstract}
We present $H$-band $(1.65 \mu \mathrm{m})$ imaging of 23 low redshift $(z \leq 0.3)$ BL Lac objects that were previously investigated by us in the optical $R$-band. For all the observed objects, the host galaxy is clearly detected and well represented by an elliptical model, with average scale length $R(\mathrm{e})=7.2 \pm 3.6 \mathrm{kpc}$ and average absolute magnitude $M(\mathrm{H})=-25.9 \pm 0.6$. BL Lac hosts are therefore luminous (massive) elliptical galaxies indistinguishable from those of low redshift radio galaxies and inactive ellipticals. The best-fit $H$-band Kormendy relation of $\mu_{\mathrm{e}}=3.7 \log r_{\mathrm{e}}(\mathrm{kpc})+14.8 \mathrm{mag} \mathrm{arcsec}^{-2}$ is in agreement with that obtained for normal ellipticals and radio galaxies. This structural and dynamical similarity indicates that all massive elliptical galaxies can experience nuclear activity without significant perturbation of their global structure.

The new observations are combined with literature data (Kotilainen et al. 1998; Scarpa et al. 2000b; Cheung et al. 2003) of 18 objects in order to construct a sizeable dataset of 41 BL Lacs with determined host characteristics in both optical and NIR. This extended sample is used to investigate the optical-NIR colour and colour gradient properties of the host galaxies of BL Lacs and to perform a direct comparison with other elliptical galaxies with and without nuclear activity.

The integrated optical/near-infrared colour $(R-H=2.2 \pm 0.4)$ and colour gradient $(\Delta(R-H) / \Delta(\log r)=-0.38 \pm 0.60)$ distributions of the BL Lac hosts are much wider than those for normal ellipticals with old stellar populations, and many BL Lacs have bluer hosts and/or steeper colour gradients than those in normal ellipticals. The blue colours are most likely caused by a young stellar population, and indicates a link between star formation caused by an interaction/merging event and the onset of the nuclear activity. However, the lack of obvious signs of interaction may require a significant time delay between the event with associated SF episodes and the start of the nuclear activity.
\end{abstract}

Key words. galaxies: BL Lacertae objects: general - galaxies: active - galaxies: elliptical and lenticular, cD galaxies: nuclei - galaxies: photometry - infrared: galaxies

\section{Introduction}

BL Lac objects are active galactic nuclei (AGN) characterized by luminous, rapidly variable UV-to-near-infrared (NIR) non-thermal continuum emission and polarization, strong compact flat spectrum radio emission and superluminal motion. Similar properties are observed also in flat spectrum radio quasars (FSRQ) and the two types of AGN are often grouped together in the blazars class. The clearest difference between BL Lacs and FSRQs is that the latter have strong broad emission lines of similar strength to normal quasars, while in BL Lacs they are very weak or absent (e.g. Scarpa \& Falomo 1997).

These extreme properties are commonly interpreted assuming that the observed emission is dominated by a relativistically beamed synchrotron jet oriented close to our line-of-sight (Blandford \& Rees 1978; Urry \& Padovani 1995). This model

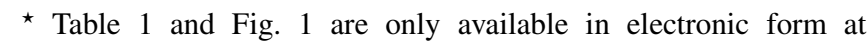
http://www. edpsciences.org implies the existence of a more numerous population of intrinsically identical objects but with their jet oriented at larger angles to our line-of-sight. In the current unified models for radio-loud AGN (e.g. Urry \& Padovani 1995), BL Lacs are low luminosity core-dominated (FR I) radio galaxies (RG) with jets pointing close to our line-of-sight, while FSRQs are high luminosity lobe-dominated (FR II) RGs. As a consequence of this model, any orientation-independent properties of the BL Lac objects (e.g. extended radio emission, host galaxies and environments) should be identical to those of the parent population (i.e. FR I RGs).

Optical imaging studies of BL Lacs secured either with groundbased telescopes (e.g. Abraham et al. 1991; Stickel et al. 1993; Falomo 1996; Wurtz et al. 1996; Falomo \& Kotilainen 1999; Pursimo et al. 2002; Nilsson et al. 2003) or with the HST (e.g. Falomo et al. 1997b; Jannuzi et al. 1997; Scarpa et al. 2000a; Urry et al. 2000) have shown that virtually all nearby $(z<0.5)$ objects are hosted in giant ellipticals with, in some cases, complex morphology (close companions, jets). 
They have similar magnitude to both FR I and FR II RGs (Govoni et al. 2000). Although some claims of a significant disc component have been made in some sources (Stocke et al. 1995; Wurtz et al. 1996), the HST imaging survey of BL Lacs found overwhelming preference for elliptical hosts (Scarpa et al. 2000a; Falomo et al. 2000). Taken together, these works have demonstrated that the hosts of (virtually) all BL Lacs are luminous (mostly unperturbed) elliptical galaxies located in poor environments. Both the global (Urry et al. 2000) and the detailed (e.g. ellipticity, isophote twisting and shape) characteristics (Falomo et al. 2000) of the host galaxies are indistinguishable from those of inactive massive ellipticals.

On the other hand, because most of these studies have been performed in only one (usually optical) band, little colour information of BL Lacs exists. NIR imaging of small $(<10$ sources) BL Lac samples were performed by Kotilainen et al. (1998; hereafter K98), Scarpa et al. (2000b; hereafter S00) and Cheung et al. (2003; hereafter C03). These studies suggested that the optical-NIR colour and the colour gradient of BL Lac hosts is similar to those of inactive ellipticals (e.g. Peletier et al. 1990) and low redshift RGs (e.g. de Vries et al. 1998).

The existing dataset therefore suggests that the nuclear activity does not strongly affect the properties of BL Lac hosts, which can be explained by the bulk of star formation (SF) occurring at high redshift, followed by passive stellar evolution (Stanford et al. 1998). Indications of a very old, coeval stellar population have been found for RGs and inactive ellipticals (e.g. McCarthy 1993). For example, galaxy interactions and mergers, often invoked to explain AGN phenomena, do not seem to induce massive SF in BL Lac hosts, possibly due to low gas density and/or different timescales of SF and nuclear activity. This result has obvious implications for the host galaxy-AGN connection and the evolution of AGN. For example, since many inactive ellipticals appear to have supermassive black holes (e.g. van der Marel 1999), it is possible that all ellipticals have the potential to become active with increased accretion.

The colour properties of Bl Lac hosts are, however, based on optical/NIR imaging of only 20 objects that were investigated by different groups (seven sources in K98, ten in S00, eight in $\mathrm{C} 03$; five common objects among the samples), and need to be confirmed using a larger homogeneous sample. In this paper, we present deep high spatial resolution NIR $H$-band $(1.65 \mu \mathrm{m})$ images of 23 low redshift $(z<0.3)$ BL Lac objects (19 high-frequency-peaked and 4 low-frequency-peaked objects) aimed at deriving the NIR properties of their host galaxies. There are two objects in common with the previous studies (MS $0158.5+0019$ by C03, and PKS 2254+074 by K98).

The new observations are combined with previous data in order to form a sizeable sample of 41 low redshift BL Lac objects with determined NIR and optical host galaxy properties. For all the observed objects we were able to study the host galaxy in the optical ( $R$-band) using NOT (Falomo \& Kotilainen 1999) and/or HST (Urry et al. 2000). This enables us to homogeneously investigate the integrated $R-H$ colour and its gradient for the BL Lacs and to compare them with those of RGs and inactive ellipticals. We emphasize that still few colour gradient measurements exist for any class of AGN, and measuring them in BL Lacs will find immediate implications to studies of other types of AGN.

In Sect. 2, we describe the observations, data reduction and the method of the analysis. Our results are presented in Sect. 3 and conclusions in Sect. 4. Throughout this paper, $H_{0}=$ $50 \mathrm{~km} \mathrm{~s}^{-1} \mathrm{Mpc}^{-1}$ and $\Omega_{0}=0$ are used.

\section{Observations, data reduction and modeling of the luminosity profiles}

The observations were carried out at the $2.5 \mathrm{~m}$ Nordic Optical Telescope (NOT) on four nights in July 2002, using the $1024 \times$ 1024 px NOTCam NIR camera with pixel scale $0.235^{\prime \prime} \mathrm{px}^{-1}$, giving a field of view of $4 \times 4 \mathrm{arcmin}^{2}$. The $H$-band $(1.65 \mu \mathrm{m})$, corresponding to the minimum in the nucleus/host luminosity ratio for low $z$ objects, was used for all the observations. The seeing during the observations was excellent, ranging from 0.'5 to $0 ! 9 F W H M$ (average and median =0!'7). A journal of the observations is given in Table 1. For the majority of the objects the images were acquired keeping the target in the field by dithering it across the array and acquiring several short exposures at each position. Individual exposures were then coadded to achieve the final integration time (see Table 1). Only for the most nearby targets, object frames were interspersed with sky frames in order to perform an accurate sky subtraction.

Data reduction was performed using the NOAO Image Reduction and Analysis Facility $\left(\mathrm{IRAF}^{1}\right)$. Bad pixels were identified via a mask made from the ratio of two sky flats with different illumination level, and were substituted by interpolating across neighboring pixels. Sky subtraction was obtained using a median averaged sky frame (for nearby sources) or a median averaged frame of the temporally closest frames (for most sources). Flat-fielding was made using twilight sky frames, and images of the same target were registered and combined using field stars as reference points to obtain the final reduced coadded image.

Observations of standard stars taken from Hunt et al. (1998), were used for the photometric calibration, for which we estimate an accuracy of $\leq 0.1 \mathrm{mag}$. No K-correction was applied to the host galaxy magnitudes as the size of this correction is insignificant at $z \leq 0.3$ in the $H$-band $(\Delta m(H)<0.02$; Neugebauer et al. (1985). No K-correction was applied to the nuclear component, assumed to have a power-law spectrum $\left(f_{v} \propto v^{-\alpha}\right)$ with $\alpha \sim 1$. The interstellar extinction corrections were computed using $R$-band extinction coefficient from Urry et al. (2000) and $A_{H} / A_{R}=0.234$ (Cardelli et al. 1989).

In order to characterize the properties of the host galaxies, azimuthally averaged one-dimensional radial luminosity profiles were extracted for each BL Lac object and for various stars in the frames down to surface brightness of $\mu(H) \sim$ $23 \mathrm{mag} \operatorname{arcsec}^{-2}$. As all the sample objects are well resolved and almost all are known from optical studies to have quite

\footnotetext{
IRAF is distributed by the National Optical Astronomy Observatories, which are operated by the Association of Universities for Research in Astronomy, Inc., under cooperative agreement with the National Science Foundation.
} 
Table 2. Properties of the host galaxies ${ }^{a}$.

\begin{tabular}{|c|c|c|c|c|c|c|c|c|c|c|}
\hline (1) & (2) & $\begin{array}{l}A_{H}{ }^{b} \\
\text { mag } \\
(3)\end{array}$ & $m_{H}($ nuc $)$ & $m_{H}(\mathrm{gal})$ & $\mu_{\mathrm{e}}^{c}$ & $\begin{array}{l}r_{\mathrm{e}} \\
\operatorname{arcsec} \\
(7)\end{array}$ & $\begin{array}{r}R_{\mathrm{e}} \\
\mathrm{kpc} \\
(8)\end{array}$ & $M_{H}(\mathrm{nuc})$ & $M_{H}(\mathrm{gal})$ & $N / G$ \\
\hline 1ES $0120+340$ & 0.272 & 0.06 & 14.57 & 14.32 & 17.44 & 2.30 & 12.8 & -26.77 & -27.19 & 0.68 \\
\hline MS 0158.5+0019 & 0.298 & 0.03 & 16.54 & 15.67 & 18.41 & 2.10 & 12.4 & -25.02 & -26.02 & 0.40 \\
\hline MS 0205.7+3509 & 0.318 & 0.07 & 16.15 & 16.76 & 17.53 & 0.90 & 5.6 & -25.57 & -25.09 & 1.6 \\
\hline MS $0317.0+1834$ & 0.190 & 0.11 & 15.58 & 15.16 & 18.06 & 1.60 & 6.8 & -24.90 & -25.52 & 0.56 \\
\hline Mrk 180 & 0.045 & 0.02 & 13.71 & 11.86 & 18.04 & 4.30 & 5.3 & -23.50 & -25.60 & 0.14 \\
\hline 1ES $1218+304$ & 0.182 & 0.02 & 14.51 & 14.29 & 18.72 & 3.15 & 13.0 & -25.87 & -26.26 & 0.70 \\
\hline MS $1221.8+2452$ & 0.218 & 0.02 & 14.69 & 15.83 & 16.01 & 0.50 & 2.4 & -26.12 & -25.09 & 2.6 \\
\hline MS $1229.2+6430$ & 0.164 & 0.02 & 18.32 & 15.06 & 18.36 & 1.75 & 6.7 & -21.82 & -25.23 & 0.04 \\
\hline 1ES $1255+244$ & 0.140 & 0.01 & 15.91 & 14.44 & 18.69 & 2.50 & 8.4 & -23.86 & -25.54 & 0.21 \\
\hline 1ES $1426+428$ & 0.129 & 0.02 & 15.37 & 13.45 & 18.57 & 3.60 & 11.3 & -24.21 & -26.21 & 0.16 \\
\hline 1ES $1440+122$ & 0.162 & 0.02 & 16.23 & 14.15 & 18.87 & 3.35 & 12.6 & -23.88 & -26.14 & 0.12 \\
\hline MS $1458.8+2249$ & 0.235 & 0.04 & 14.21 & 15.18 & 17.89 & 1.70 & 8.5 & -26.78 & -25.95 & 2.1 \\
\hline MS $1552.1+2020$ & 0.273 & 0.04 & 15.83 & 14.42 & 17.88 & 2.70 & 15.0 & -25.52 & -27.09 & 0.24 \\
\hline Mrk 501 & 0.034 & 0.02 & 12.22 & 10.41 & 17.13 & 5.30 & 5.0 & -24.36 & -26.55 & 0.13 \\
\hline I Zw 187 & 0.055 & 0.03 & 14.44 & 13.19 & 18.51 & 3.00 & 4.4 & -23.21 & -24.68 & 0.26 \\
\hline 3C 371 & 0.051 & 0.05 & 12.53 & 11.97 & 16.59 & 2.15 & 3.0 & -24.95 & -25.89 & 0.42 \\
\hline 1ES $1853+671$ & 0.212 & 0.06 & 16.87 & 15.44 & 19.40 & 2.80 & 13.0 & -23.87 & -25.47 & 0.23 \\
\hline 1ES 1959+650 & 0.048 & 0.11 & 13.69 & 12.30 & 18.27 & 3.95 & 5.1 & -23.66 & -25.17 & 0.25 \\
\hline BL Lac & 0.069 & 0.21 & 11.90 & 12.23 & 15.05 & 1.00 & 1.8 & -26.26 & -26.00 & 1.3 \\
\hline PKS 2201+04 & 0.027 & 0.06 & 15.50 & 11.64 & 17.39 & 3.30 & 2.5 & -20.58 & -24.68 & 0.02 \\
\hline PKS 2254+074 & 0.194 & 0.06 & 13.96 & 14.16 & 18.43 & 3.05 & 13.2 & -26.57 & -26.52 & 1.0 \\
\hline 1ES $2326+174$ & 0.213 & 0.04 & 17.12 & 14.75 & 17.20 & 1.40 & 6.5 & -23.63 & -26.16 & 0.10 \\
\hline 1ES $2344+514$ & 0.044 & 0.17 & 15.69 & 11.60 & 17.15 & 3.20 & 3.8 & -21.46 & -25.86 & 0.02 \\
\hline
\end{tabular}

${ }^{a}$ Columns 1 and 2 give the name and redshift of the object; Col. 3: the interstellar extinction correction; Cols. 4 and 5: the apparent nuclear and host galaxy magnitude; Col. 6: the surface brightness $\mu$ (e); Cols. 7 and 8: the bulge scalelength in arcsec and kpc; Cols. 9 and 10: the absolute nuclear and host galaxy magnitude; and Col. 11: the nucleus/galaxy luminosity ratio.

${ }^{b}$ The interstellar extinction corrections were computed using $R$-band extinction coefficient from Urry et al. (2000) and $A_{H} / A_{R}=0.234($ Cardelli et al. 1989).

${ }^{c}$ Corrected for galactic extinction and cosmological dimming.

round and undisturbed host galaxies (Falomo \& Kotilainen 1999; Urry et al. 2000), we are confident that the 1D modelling is not affected by unusual features in the host galaxy. Note that the most problematic case in this respect is $1 \mathrm{ES} 1440+122$, which has a companion galaxy at the redshift of the BL Lac. They are undergoing weak interaction that is likely responsible for the slightly boxy isophotes of 1ES 1440+122 (Falomo et al. 2000) and the slight isophotal twisting of the companion (Heidt et al. 1999). There is, however, no evidence for disturbed morphology of 1ES $1440+122$ in the 2D HST analysis (Falomo et al. 2000) and therefore we assume that the BL Lac host is unperturbed even in this case.

The luminosity profiles were decomposed into the point source and galaxy components by an iterative least-squares fit to the observed profile. We fit the data using both elliptical $r^{1 / 4}$ (de Vaucouleurs law) and exponential disc models to represent the host galaxy. However, and consistently with the results from previous optical and NIR studies, in no case did the disc model give a better fit than the elliptical one. We estimate the uncertainty of the derived host galaxy magnitudes to be $\sim \pm 0.2 \mathrm{mag}$.

\section{Results and discussion}

In Fig. 1 we show the $H$-band contour plots of all the observed BL Lac objects. We are able to clearly detect the host galaxy in all the observed targets. In Fig. 2, we show the azimuthally averaged $H$-band radial luminosity profiles of each BL Lac object, together with the best-fit models overlaid. The results of the best-fit modelling are summarized in Table 2 .

Three host galaxies in the sample were previously studied in the NIR (MS 0158.5+0019 studied by C03, and 

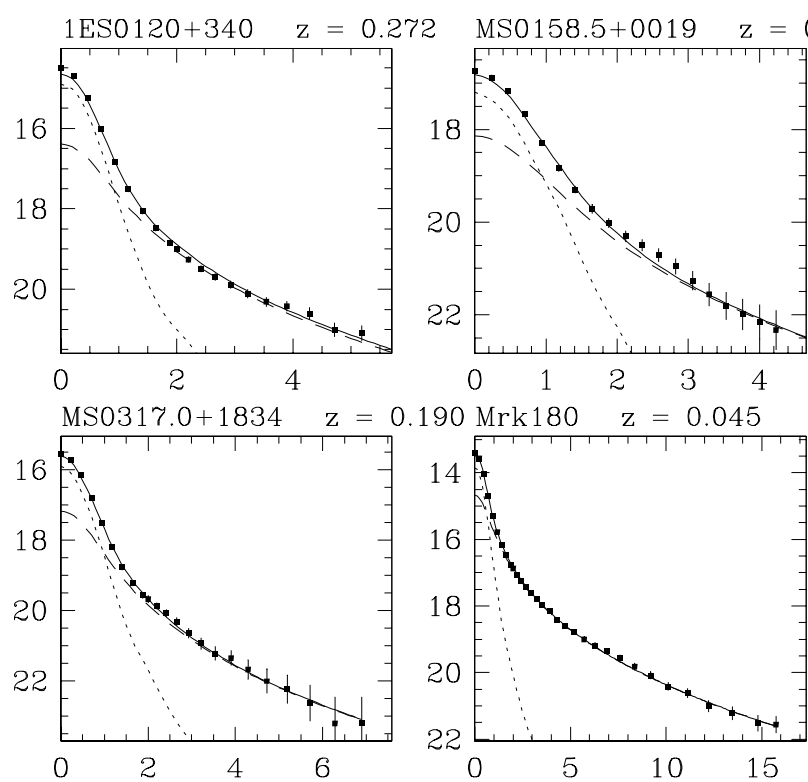

$190 \operatorname{Mrk} 180 \quad \mathrm{z}=0.045$
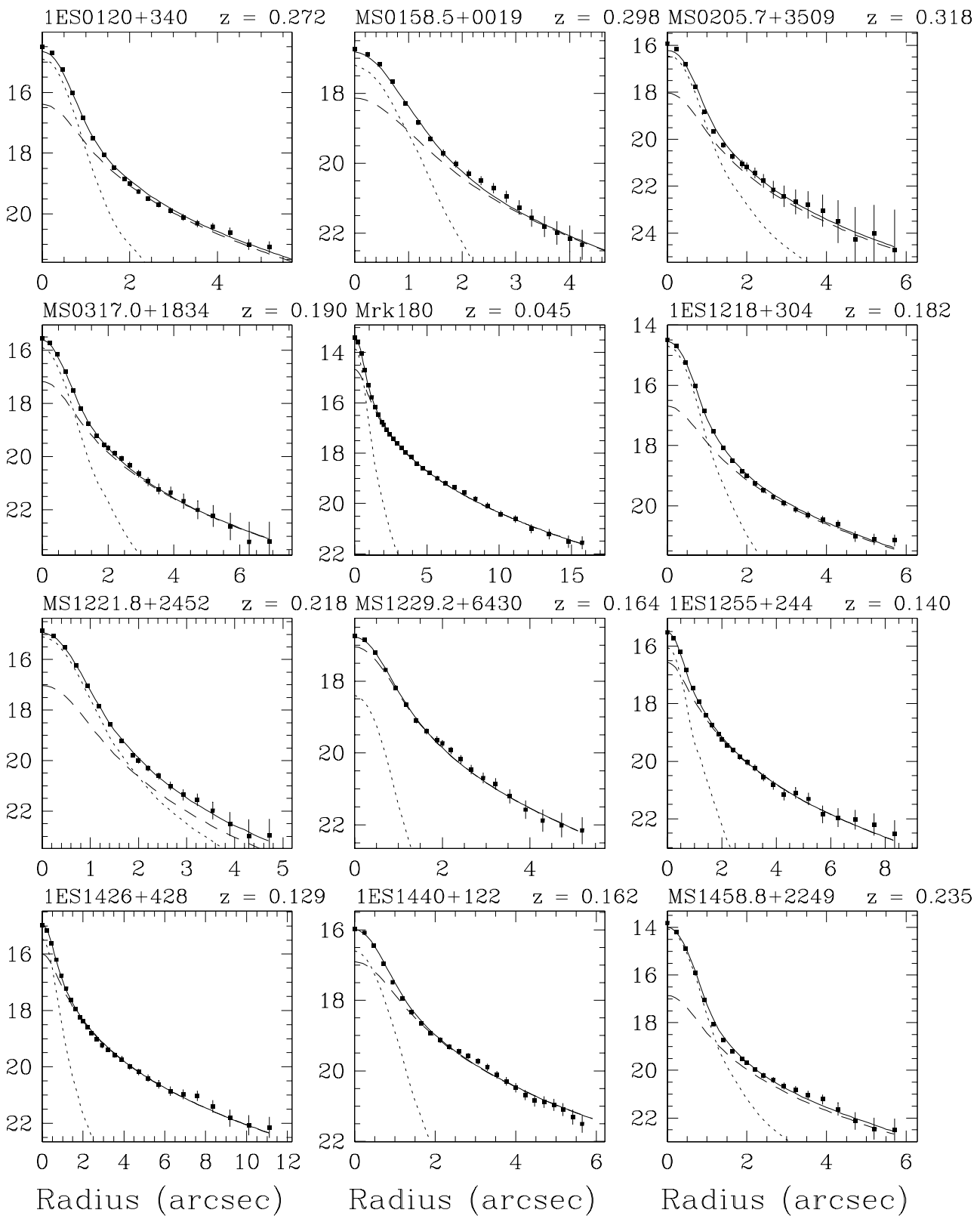

Fig. 2. The observed $H$-band azimuthally averaged radial surface brightness profile (solid points with error bars) for each BL Lac object, overlaid with the scaled PSF model (dotted line), the de Vaucouleurs $r^{1 / 4}$ model convolved with the PSF (dashed line), and the fitted PSF + host model profile (solid line). The $y$-axis is plotted in mag $\operatorname{arcsec}^{-2}$.

PKS 2254+074 studied by K98; MS 0317.0+1834 by Wright et al. 1998). The comparison between these studies is presented in Table 3. For MS 0158.5+0019, the derived host magnitudes are in good agreement, whereas we find larger bulge scale length. C03 find much redder optical-NIR colour and much steeper colour gradient than in this work. PKS 2254+074, already studied by K98, was re-observed here because K98 obtained only a short exposure (10 $\mathrm{min})$ under poor sky conditions and the object was situated in a bad area of the array during their observations. Consequently, the host properties were less accurately than for the other resolved BL Lacs in K98. With improved data quality, we detect a much brighter host galaxy with a more reasonable bulge scale length, slightly redder colour and steeper colour gradient. Finally, MS 0317.0+1834 was studied with poorer quality data in the $K$-band by Wright et al. (1998), who marginally resolved somewhat fainter and a factor of two larger host than what is found in this work.

\subsection{Morphology and luminosity of the host galaxies}

In Fig. 3, we show the $H-\mathrm{z}$ (Hubble) diagram for the BL Lac hosts (this work, K98, S00 and C03), compared with the established relation for RGs (solid line; Willott et al. 2003). The BL Lac hosts follow the same trend in the $\mathrm{H}-\mathrm{z}$ diagram as that traced by RGs, although there is a tendency for the BL Lac hosts to be on average fainter than the RG sample. The same trend is visible in Fig. 4, where we report the $H$-band host galaxy absolute magnitude vs. redshift for the BL Lacs 

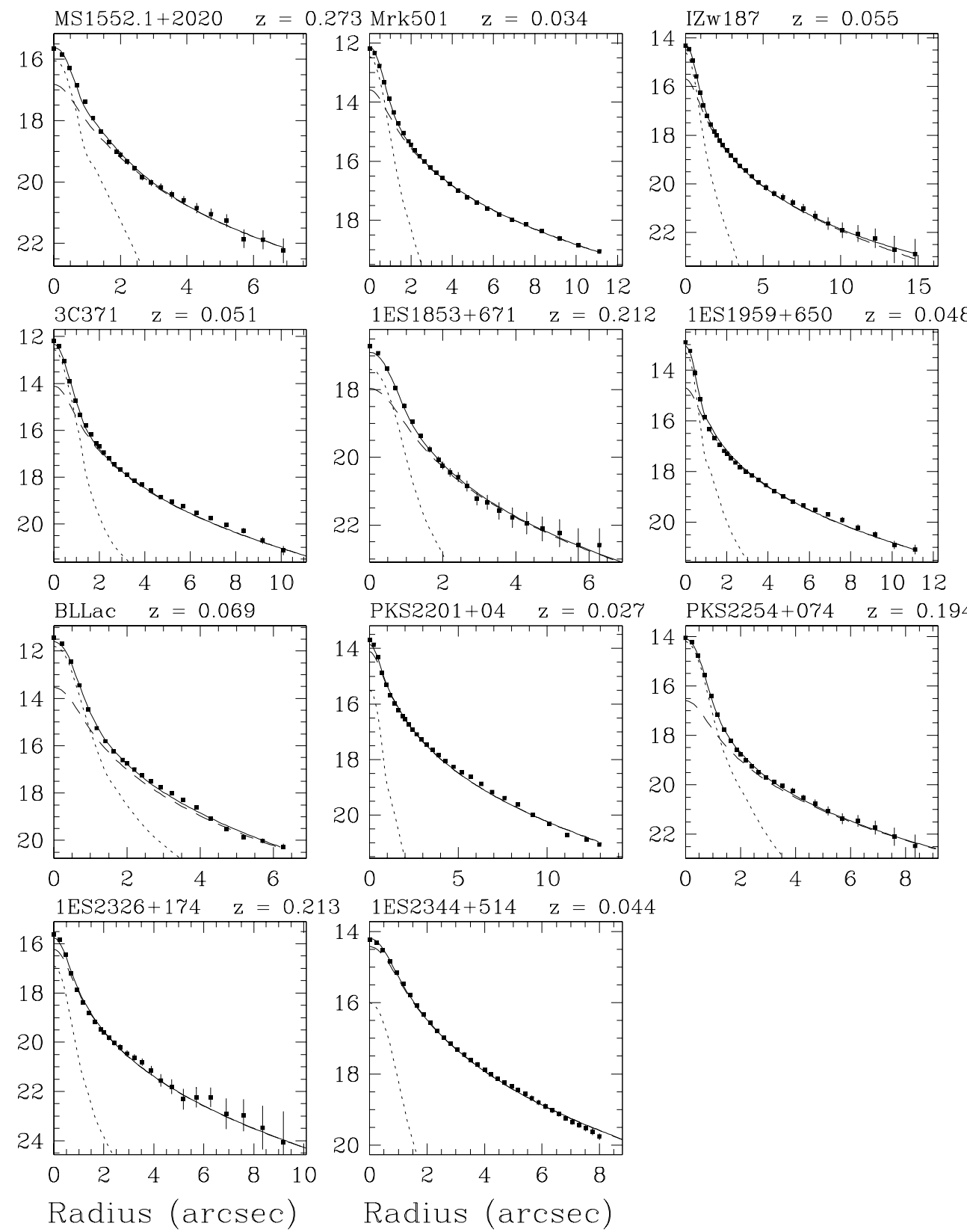

Fig. 2. continued.

(this work, K98, S00 and C03) and RGs. In the NIR, most of the host galaxies of low redshift BL Lacs are encompassed within $\mathrm{M}^{*}$ and $\mathrm{M}^{*}-2$ (where an $\mathrm{M}^{*}$ galaxy has $M(H)=-25.0 \pm$ 0.3; Mobasher et al. 1993), in agreement with previous results derived in the optical (e.g. Falomo \& Kotilainen 1999; Urry et al. 2000).

The comparison among the various samples of the average NIR absolute magnitudes of the host galaxies is given in Table 4 . These samples span a moderately large range in redshift from $z \sim 0.03$ up to $z \sim 0.3$. All magnitudes were extinction-corrected and transformed into our adopted cosmology and into the $H$-band, assuming the average colour of giant ellipticals, $H-K=0.22$ (Recillas-Cruz et al. 1990). The average absolute magnitude of the low redshift $(z \leq 0.3)$ BL Lac hosts in this work is $M(H)=-25.8 \pm 0.7$, indistinguishable from that of the other BL Lac samples. For the total combined sample of 41 BL Lacs, we obtain average $M(H)=-25.9 \pm 0.6$. As found in the optical by e.g. Wurtz et al. (1996), Falomo \& Kotilainen (1999) and Urry et al. (2000), BL Lac hosts have slightly lower luminosities in the NIR than nearby brightest cluster member galaxies $(\mathrm{BCM} ; M(H)=-26.3 \pm 0.3$; Thuan \& Puschell 1989) and the (mainly 3C) RGs at $z<0.3$ studied by Willott et al. (2003).

The average bulge scalelength of the 23 low redshift BL Lac hosts in this work is $R(\mathrm{e})=7.8 \pm 4.1 \mathrm{kpc},(R(\mathrm{e})=$ $7.2 \pm 3.6 \mathrm{kpc}$. for the combined sample of $41 \mathrm{BL}$ Lacs). Slightly smaller (K98 and C03) and larger (S00) values were found in previous studies (see Table 4 for details). Consistently with optical studies, therefore, the BL Lac host galaxies are with a few exceptions larger than normal ellipticals 
Table 3. Comparison with previous studies for common BL Lacs.

\begin{tabular}{llllll}
\hline \hline $\begin{array}{l}\text { Object } \\
(1)\end{array}$ & $R_{\mathrm{e}}$ & $\begin{array}{l}M_{H}(\mathrm{gal}) \\
(2)\end{array}$ & $\begin{array}{l}R-H \\
(3)\end{array}$ & $\begin{array}{l}\Delta(R-H) / \Delta(\log r) \\
(5)\end{array}$ & $\begin{array}{l}\text { Ref. } \\
(6)\end{array}$ \\
\hline MS 0158.5+0019 & 12 & -26.0 & 2.1 & -0.22 & This work \\
& 4.7 & -26.3 & 3.3 & -2.0 & C03 \\
PKS 2254+074 & 13 & -26.5 & 2.1 & -0.75 & This work \\
& 32 & -25.6 & 1.8 & 0.10 & K98 \\
MS 0317.0+1834 & 6.8 & -25.5 & & & This work \\
& 14 & -25.2 & & & Wright et al. (1998) \\
\hline
\end{tabular}

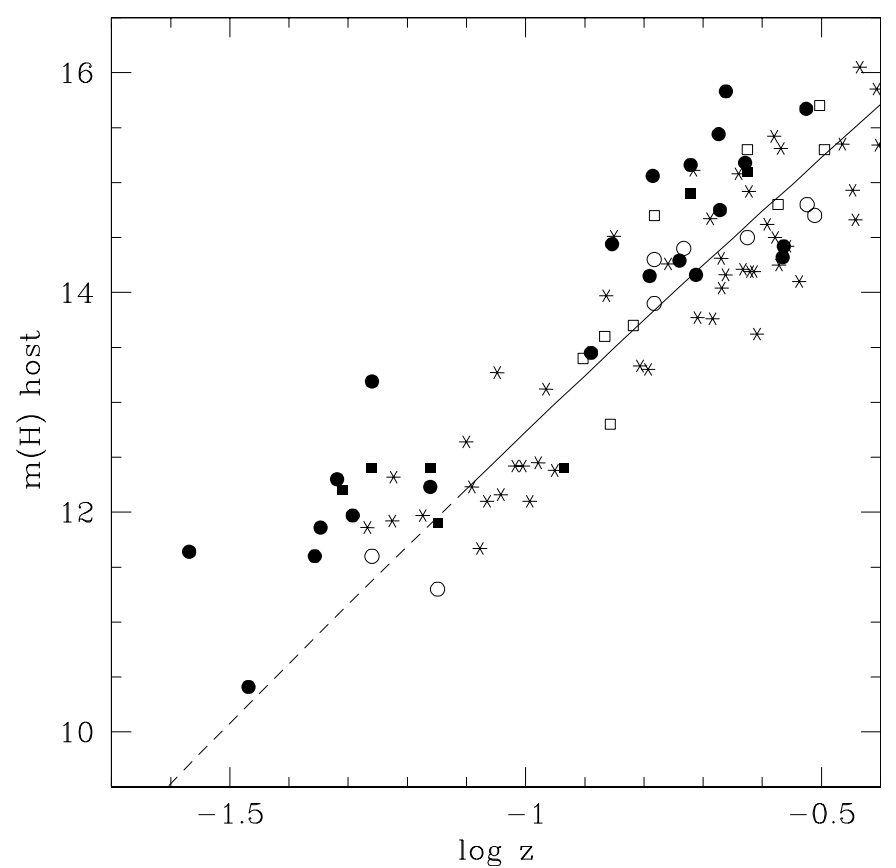

Fig. 3. Plot of the apparent $H$ magnitude of the host galaxies vs. redshift (Hubble diagram). The BL Lacs are marked as filled circles (this work), filled squares (K98), open squares (S00) and open circles (C03). The solid line is the $K-z$ relation for RGs derived by Willott et al. (2003), converted to the $H$-band assuming $H-K=0.2$. The dashed line is the extrapolation of the relation towards lower redshift. The RGs are shown as asterisks.

(e.g. Capaccioli et al. 1992), but smaller than low redshift RGs studied in the optical by Govoni et al. $(2000 ; R(\mathrm{e})=16 \pm$ $10 \mathrm{kpc})$ and in NIR by Taylor et al. $(1996 ; R(\mathrm{e})=26 \pm 16 \mathrm{kpc})$.

\subsection{Surface brightness - effective radius (Kormendy) relation}

NIR observations map the bulk of the slowly evolving stellar populations of galaxies, and are therefore ideal for investigating luminosity and galaxy morphology. We have investigated the $H$-band Kormendy relation, which is a projection of the fundamental plane, relating the effective radius, $r_{\mathrm{e}}$, to the surface brightness at that radius, $\mu_{\mathrm{e}}$ (Kormendy 1977; Djorgovski \& Davis 1987; Kormendy \& Djorgovski 1989). This relation is closely related to the morphological and dynamical structure of galaxies, and to their formation processes.

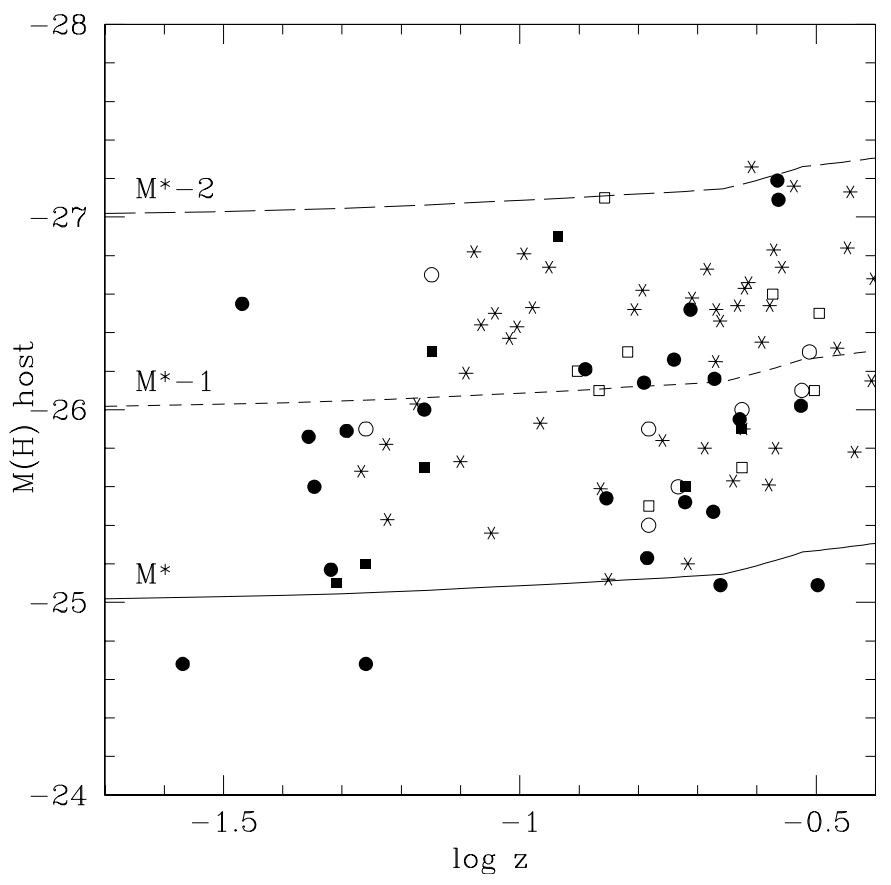

Fig. 4. Plot of the absolute $H$ band magnitude of the host galaxies vs. redshift. For symbols, see Fig. 3. The solid, short-dashed and longdashed lines are the luminosities of $L *(M(H) \sim-25.0$ at low redshift; Mobasher et al. 1993), $L *-1$ and $L *-2$ galaxies, respectively, following the passive evolution model of Bressan et al. (1998).

We combined our $\mu_{\mathrm{e}}$ and $r_{\mathrm{e}}$ data with those from K98, S00 and $\mathrm{C} 03$ to construct the Kormendy relation in the NIR for the largest available sample of BL Lacs (see Fig. 5). The effective surface brightnesses of the hosts have been corrected for galactic extinction and cosmological dimming $(10 \times \log (1+z))$. The $K$-band data from $\mathrm{C} 03$ were converted to the $H$-band assuming $H-K=0.22$ (Recillas-Cruz et al. 1990). We find a best-fit linear relation for the BL Lacs to be $\mu_{\mathrm{e}}(\mathrm{H})=$ $3.7( \pm 0.3) \log r_{\mathrm{e}}+14.8( \pm 0.3) \mathrm{mag} \operatorname{arcsec}^{-2}$. This is very similar to that derived for normal elliptical galaxies $\left(\mu_{\mathrm{e}}(\mathrm{H})=\right.$ $4.3 \log r_{\mathrm{e}}+14.3 \mathrm{mag} \operatorname{arcsec}^{-2}$; Pahre et al. 1995).

This similarity between BL Lacs and normal ellipticals (which also holds for RGs, e.g. Govoni et al. 2000) suggests that BL Lac hosts (and RGs) are dynamically very similar and occupy the same region of this projection of the fundamental plane as normal ellipticals. This indicates that the formation processes and the structure of galaxies hosting radio sources are similar to those of radio-quiet ellipticals. This result is reinforced by spectroscopic studies of the fundamental 
Table 4. Comparison of the average NIR host galaxy properties ${ }^{a}$.

\begin{tabular}{|c|c|c|c|c|c|c|}
\hline $\begin{array}{l}\text { Sample } \\
\text { (1) }\end{array}$ & $\begin{array}{l}\text { Filter } \\
\text { (2) }\end{array}$ & $\begin{array}{r}N \\
(3)\end{array}$ & $\begin{array}{l}\langle z\rangle \\
(4)\end{array}$ & $\begin{array}{l}\left\langle M_{H}(\mathrm{nuc})\right\rangle \\
(5)\end{array}$ & $\begin{array}{l}\left\langle M_{H}(\text { host })\right\rangle \\
(6)\end{array}$ & $\begin{array}{l}\langle R(\mathrm{e})\rangle \\
(7)\end{array}$ \\
\hline BL (this work) & $H$ & 23 & $0.155 \pm 0.091$ & $-24.5 \pm 1.6$ & $-25.8 \pm 0.7$ & $7.8 \pm 4.1$ \\
\hline BL C03 & $K$ & 8 & $0.186 \pm 0.088$ & $-25.6 \pm 0.8$ & $-26.0 \pm 0.4$ & $4.2 \pm 2.3$ \\
\hline BL S00 & $H$ & 9 & $0.206 \pm 0.074$ & $-25.0 \pm 1.6$ & $-26.2 \pm 0.4$ & $10 \pm 5$ \\
\hline BL K98 & $H$ & 7 & $0.112 \pm 0.068$ & $-25.7 \pm 1.7$ & $-25.8 \pm 0.5$ & $4.8 \pm 1.9$ \\
\hline BL all & $H / K$ & 42 & $0.164 \pm 0.084$ & $-25.0 \pm 1.5$ & $-25.9 \pm 0.6$ & $7.2 \pm 3.6$ \\
\hline$L^{*}$ Mobasher et al. (1993) & $K$ & 136 & $0.077 \pm 0.030$ & & $-25.0 \pm 0.2$ & \\
\hline BCM Thuan \& Puschell (1989) & $H$ & 84 & $0.074 \pm 0.026$ & & $-26.3 \pm 0.3$ & \\
\hline BCM Aragon-Salamanca et al. (1998) & $K$ & 25 & $0.449 \pm 0.266$ & & $-27.0 \pm 0.3$ & \\
\hline RG FR II Taylor et al. (1996) & $K$ & 12 & $0.214 \pm 0.049$ & $-25.1 \pm 0.7$ & $-26.1 \pm 0.8$ & $26 \pm 16$ \\
\hline RG Willott et al. (2003) $z<0.3$ & $K$ & 42 & $0.170 \pm 0.075$ & & $-26.2 \pm 0.5$ & \\
\hline
\end{tabular}

${ }^{a}$ Column (1) gives the sample; Col. 2: the filter; Col. 3: the number of objects in the sample; Col. 4: the average redshift of the sample; Cols. 5 and 6: the average $H$ band nuclear and host galaxy absolute magnitude of the sample; and Col. 7: the average $H$-band bulge scale length of the sample.

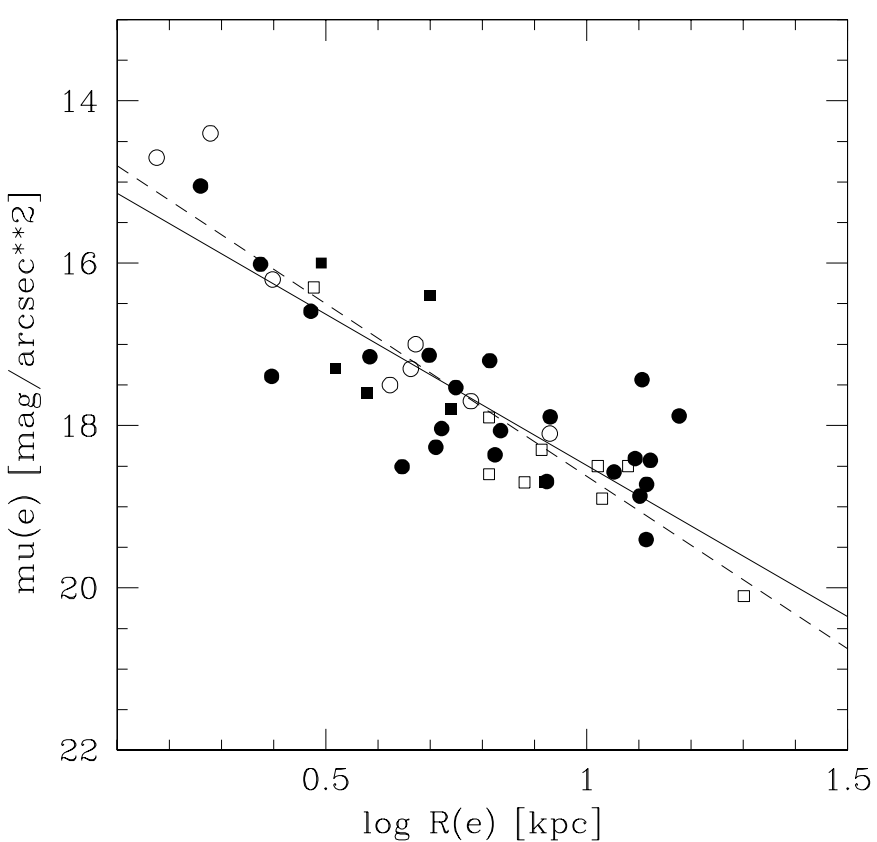

Fig. 5. The $H$-band $\mu_{\mathrm{e}}-r_{\mathrm{e}}$ relation for the full sample of BL Lac host galaxies. For symbols, see Fig. 3. The solid and dashed lines are the linear least-square best-fit relations for the BL Lacs and for normal inactive ellipticals (Pahre et al. 1995), respectively.

plane (Bettoni et al. 2001; Barth et al. 2003; Falomo et al. 2002; Falomo et al. 2003) and supports the picture in which all massive elliptical galaxies have the potential to experience a phase of nuclear activity with little influence on their global structure.

\subsection{Host galaxy colours}

The host galaxies of all the low redshift BL Lacs in our sample were previously studied by us in the optical (Falomo et al. 1997a,b; Falomo \& Kotilainen 1999; Urry et al. 2000). The new observations for 21 objects represent the first study of them

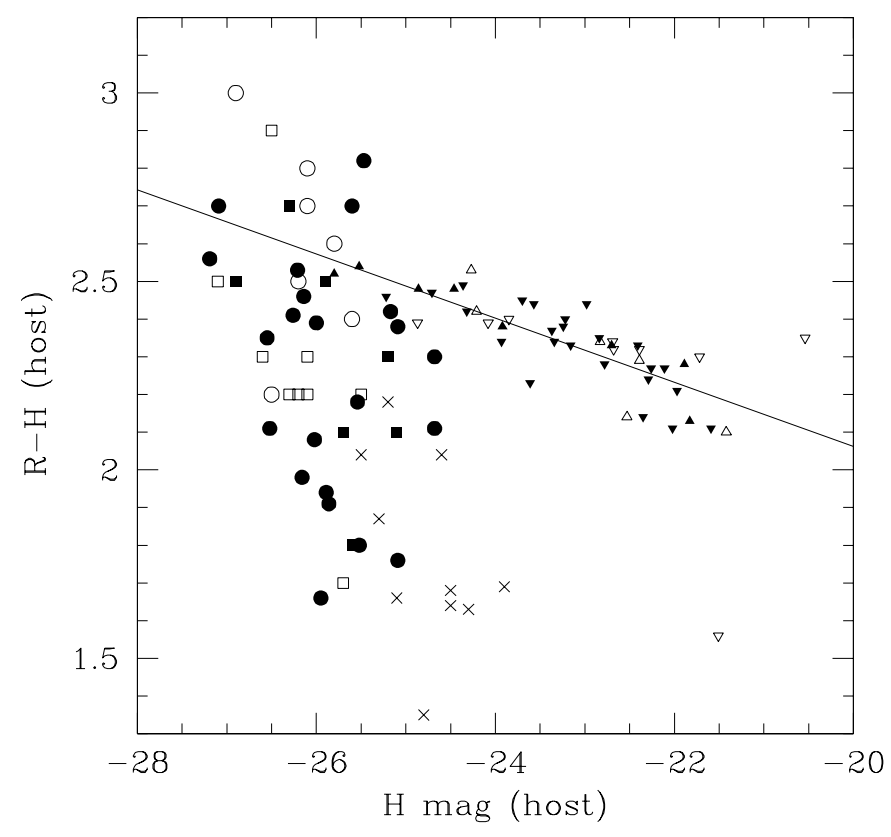

Fig. 6. The $R-H$ vs. H colour-magnitude diagram for the BL Lac host galaxies. For symbols, see Fig. 3. The other symbols indicate low redshift $(z<0.2)$ elliptical quasar hosts (crosses; from Jahnke et al. 2003) and elliptical galaxies (filled triangles) and S0 galaxies (open triangles) in the Virgo cluster, and elliptical galaxies (filled inverted triangles) and S0 galaxies (open inverted triangles) in the Coma cluster (from Bower et al. 1992a,b). The solid line shows the best-fit regression line for Virgo and Coma clusters (Bower et al. 1992b). The $V-K$ vs. V diagram of Bower et al. (1992b) has been transformed into $R-H$ vs. $\mathrm{H}$ assuming $V-R=0.6, H-K=0.2$ (see text), and distance moduli for Virgo and Coma clusters $m-M=31.0$ and 34.6, respectively (Bower et al. 1992b).

performed in the NIR. These data, combined with other NIR and optical data, allow us to address the issue of the opticalNIR colour of the BL Lac host galaxies using a sizeable and homogeneous dataset. The integrated rest-frame colours of the 
Table 5. Optical-NIR colours of the host galaxies ${ }^{a}$.

\begin{tabular}{llllllr}
\hline \hline Name & $z$ & $M(H)$ & $M(R)$ & Ref. $^{b}$ & $R-H$ & $\Delta(R-H) / \Delta(\log r)$ \\
$(1)$ & $(2)$ & $(3)$ & $(4)$ & $(5)$ & $(6)$ & -0.66 \\
\hline 1ES 0120+340 & 0.272 & -27.2 & -24.6 & 1 & 2.6 & -0.22 \\
MS 0158.5+0019 & 0.298 & -26.0 & -23.9 & 1 & 2.1 & -0.52 \\
MS 0205.7+3509 & 0.318 & -25.1 & -23.3 & 1 & 1.8 & 0.29 \\
MS 0317.0+1834 & 0.190 & -25.5 & -23.7 & 1 & 1.8 & 0.33 \\
Mrk 180 & 0.045 & -25.6 & -22.9 & 2 & 2.7 & 0.11 \\
1ES 1218+304 & 0.182 & -26.3 & -23.8 & 1 & 2.5 & -0.78 \\
MS 1221.8+2452 & 0.218 & -25.1 & -22.7 & 1 & 2.4 & -0.77 \\
MS 1229.2+6430 & 0.164 & -25.2 & -24.3 & 1 & $(0.9)$ & 0.20 \\
1ES 1255+244 & 0.140 & -25.5 & -23.4 & 1 & 2.1 & 0.38 \\
1ES 1426+428 & 0.129 & -26.2 & -23.7 & 2 & 2.5 & -0.32 \\
1ES 1440+122 & 0.162 & -26.1 & -23.7 & 2 & 2.4 & \\
MS 1458.8+2249 & 0.235 & -26.0 & -24.3 & 1 & 1.7 & 0.04 \\
MS 1552.1+2020 & 0.273 & -27.1 & -24.4 & 1 & 2.7 & -1.23 \\
Mrk 501 & 0.034 & -26.6 & -24.2 & 2 & 2.4 & -0.21 \\
I Zw 187 & 0.055 & -24.7 & -22.4 & 2 & 2.3 & -0.20 \\
3C 371 & 0.051 & -25.9 & -24.0 & 2 & 1.9 & 0.21 \\
1ES 1853+671 & 0.212 & -25.5 & -22.6 & 1 & 2.9 & -0.12 \\
1ES 1959+650 & 0.048 & -25.2 & -22.8 & 1 & 2.4 & -2.25 \\
BL Lac & 0.069 & -26.0 & -23.6 & 2 & 2.4 & -0.90 \\
PKS 2201+04 & 0.027 & -24.7 & -22.6 & 2 & 2.1 & -0.75 \\
PKS 2254+074 & 0.194 & -26.5 & -24.4 & 2 & 2.1 & -0.34 \\
1ES 2326+174 & 0.213 & -26.2 & -24.2 & 1 & 2.0 & -0.65 \\
1ES 2344+514 & 0.044 & -25.9 & -24.0 & 1 & 1.9 & \\
\hline
\end{tabular}

${ }^{a}$ Columns (1) and (2) give the name and redshift of the BL Lac; Cols. 3 and 4: the absolute magnitude of the host galaxy in the $H$-band (this work) and $R$-band (literature values), respectively; Col. 5: the references for Col. 4; Col. 6: the $R-H$ colour of the host galaxy computed from Cols. 3 and 4; and Col. 7: the $R-H$ colour gradient of the host.

${ }^{b}$ References: 1 = Falomo \& Kotilainen (1999); 2 = Falomo \& Kotilainen (in prep.).

host galaxies are given in Table 5. The average and median integrated $R-H$ host colour of the BL Lac sample in this work is $R-H=2.2 \pm 0.4$ and $R-H=2.3$, and for the total combined sample of $41 \mathrm{BL}$ Lacs $R-H=2.3 \pm 0.4$ and $R-H=2.4$.

It is well known that the integrated colours of elliptical galaxies become bluer towards fainter magnitudes (e.g. Kodama \& Arimoto 1997, and references therein). Observations of large samples of cluster galaxies (e.g. Bower et al. 1992a,b) have also shown that this colour-magnitude $(\mathrm{C}-\mathrm{M})$ relation is virtually identical for different clusters. This relation is likely connected with the processes of galaxy formation and it may depend on the combined effects of age and metallicity of the underlying dominant stellar populations.

According to this relation, therefore, the comparison of the colour properties of BL Lac hosts and other types of ellipticals must take into account the dependence of the colour on the luminosity of the galaxy. In Fig. 6 we report the optical-NIR C-M diagram of the full sample of BL Lac host galaxies, compared with those of elliptical and S0 galaxies in the Virgo and Coma clusters (Bower et al. 1992a,b). It is immediately clear that BL Lac hosts do not follow the $\mathrm{C}-\mathrm{M}$ relation of ellipticals. At variance with normal ellipticals, the BL Lac hosts exhibit a much broader range of colours, and their colours appear to be systematically bluer than those of the corresponding (inactive) ellipticals. Especially, note the very blue tail with $R-H<$ 2.0 exhibited by eight BL Lacs in the sample. This behavior is similar to that found for low redshift RGs by Govoni et al. (2000; see their Fig. 12).

A possible explanation for this trend (as also suggested by Govoni et al. 2000) is that the C-M relation for elliptical galaxies breaks down at the highest luminosities. Unfortunately, to our knowledge there are no published optical and NIR observations of normal inactive galaxies in the luminosity range covered by the BL Lacs and therefore the comparison must be based on the extrapolation of the behavior of the C-M relation. Indeed, the BL Lac hosts (and RGs in Govoni et al.) cover preferentially the bright end of the luminosity function of ellipticals, whereas very few of the ellipticals in Fig. 6 are equally luminous (but those few exhibit markedly redder colour than the active ellipticals).

On the other hand, assuming that the $\mathrm{C}-\mathrm{M}$ relation derived for lower luminosity galaxies extends towards higher luminosity, the obvious conclusion for the blue colours is that the BL Lac hosts exhibit a signature of recent SF. The broad range of colours could then reflect differences between objects in the epoch of the last burst of SF. Objects with very blue colours would have experienced the most recent SF episode, whereas those with red colours would be consistent with a single old stellar population. In this view, therefore, the bluer colours and the broader colour distribution of BL Lac hosts and low redshift RGs could mark the signature of the connection between the event(s) that have triggered and/or fuelled the nuclear activity and triggered a young stellar population. 

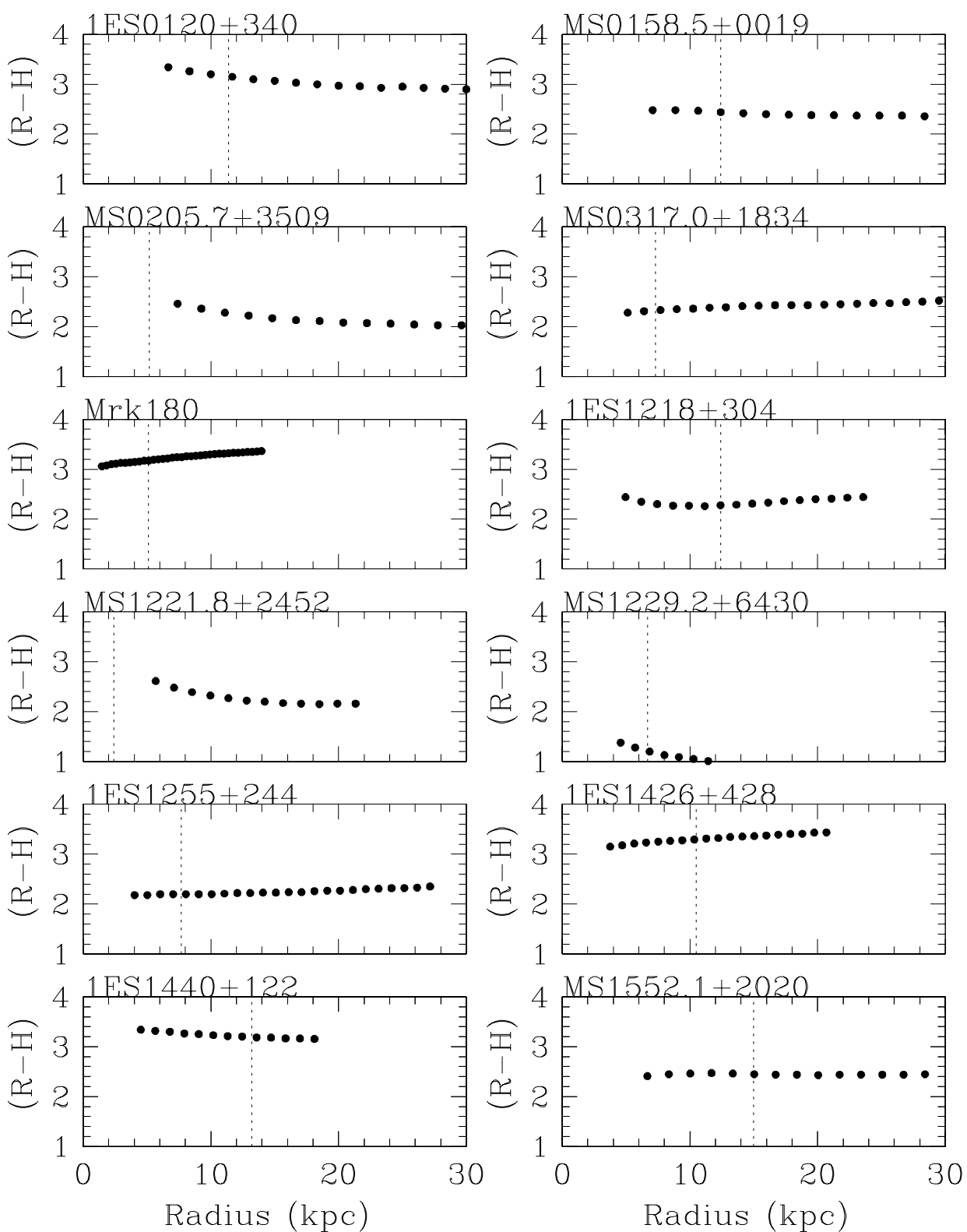

Fig. 7. The host galaxy $R-H$ radial colour profile for all the BL Lacs in the sample, calculated using the $R$-band data from Falomo \& Kotilainen (1999) and Falomo \& Kotilainen (in prep.). The dashed vertical lines refer to the effective radius derived from the fit of the $R$-band data. The profiles indicate that the host galaxies are predominantly bluer further away from the nuclei, but there are marked exceptions with bluer inner regions, e.g. MS 0317, Mrk 180 and 1ES 1853.

This scenario is corroborated by the results on the colour of low redshift $(z<0.2)$ quasar hosts obtained by Jahnke et al. (2003) (Fig. 6). For the 10 quasars hosted in elliptical galaxies they found systematically bluer colours $(R-H=1.8 \pm 0.3)$ with respect to those of inactive ellipticals, and from population synthesis modelling found for them evidence for a few Gyr stellar population, much younger than expected in old evolved elipticals. Similarly blue host colours were found for low redshift, intermediate luminosity AGN by Schade et al. (2000).

\subsection{Colour gradients}

It is well known that nearby inactive ellipticals have negative colour gradients in the sense that the galaxies become bluer with increasing radius (e.g. Peletier et al. 1990). This observed colour gradient has been ascribed to the effect of either age or metallicity gradients (e.g. Wise \& Silva 1996). Both effects can produce a colour gradient, although the latter appears to agree better with the observed gradients in nearby elliptical galaxies (Tamura et al. 2000). Independently of the origin of the colour gradient, it is important to compare the colour gradient of inactive ellipticals with that of active galaxies.

For our sample of observed BL Lac objects, we computed the radial $(R-H)$ colour profiles (see Fig. 7), using the host galaxy luminosity profiles from ground-based $R$-band images (Falomo \& Kotilainen 1999; Falomo \& Kotilainen, in prep.) when available, and from HST images (Scarpa et al. 2000a) for the others. The colour profiles against logarithmic radius 

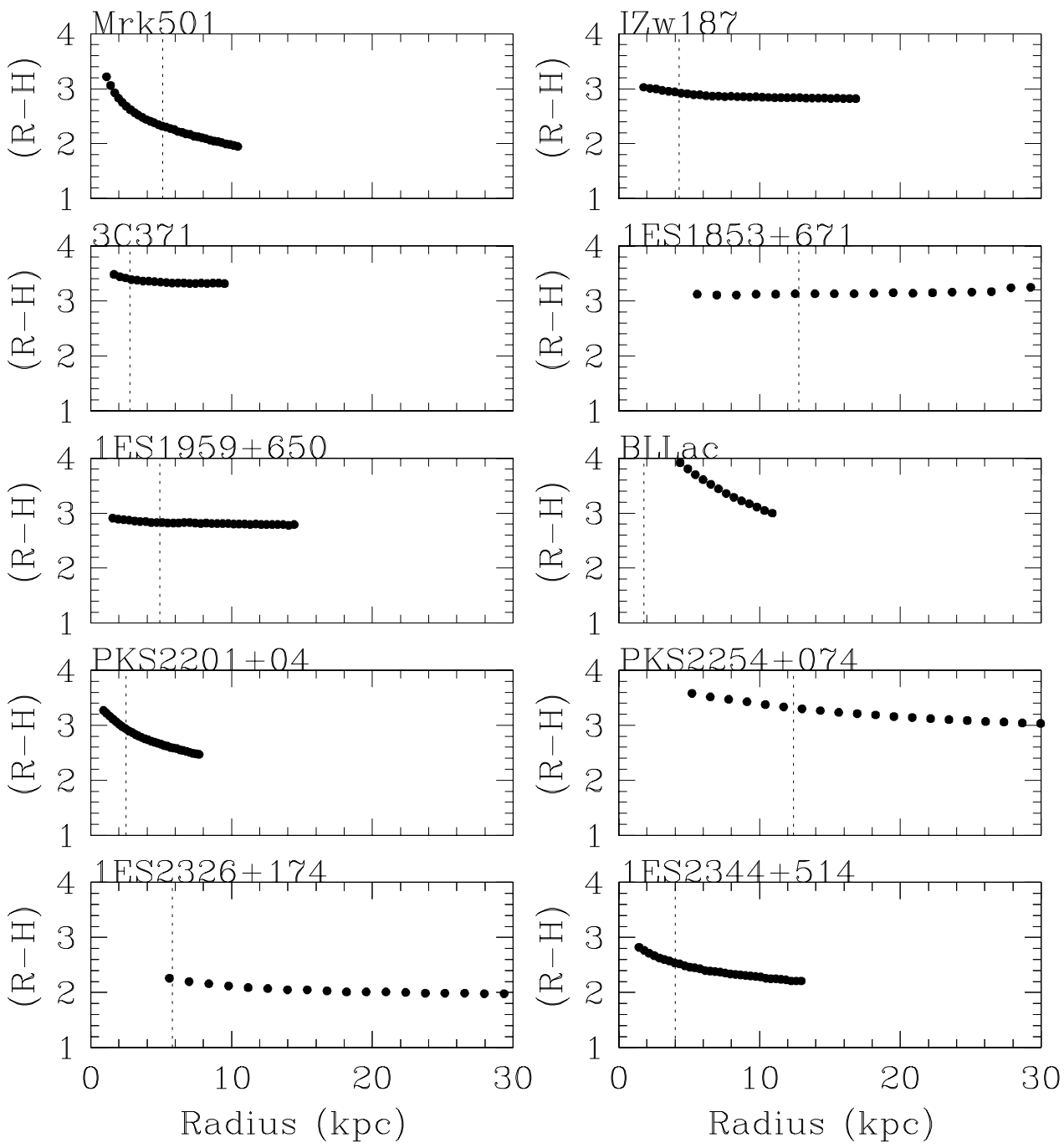

Fig. 7. continued.

are usually smooth and well represented by a linear fit. Most of the colour profiles (see Table 5) show a negative colour gradient (average $\Delta(R-H) / \Delta(\log r)=-0.38 \pm 0.60)$. This trend with similar amplitude was previously suggested by K98 and S00, but is much flatter than that of C03. In the full sample of $41 \mathrm{BL}$ Lacs, the average $\Delta(R-H) / \Delta(\log r)=-0.51 \pm$ 0.65 . However, the gradients in the $K$-band study of $\mathrm{C} 03$ $(\Delta(R-K) / \Delta \log r=-1.27 \pm 0.73)$ may be influenced by the use of small NIR effective radii (average $4.2 \mathrm{kpc}$ ), compared with $\sim 10 \mathrm{kpc}$ in the $R$-band (Falomo \& Kotilainen 1999; Urry et al. 2000). Therefore, excluding the data of $\mathrm{C} 03$, the average colour gradient in the combined sample becomes $\Delta(R-H) / \Delta(\log r)=$ $-0.34 \pm 0.50$.

The amplitude of the negative colour gradient in BL Lacs is steeper than that exhibited by normal inactive ellipticals (e.g. $\Delta(V-K) / \Delta(\log r)=-0.16 \pm 0.18$; Peletier et al. 1990 ; $\Delta(V-K) / \Delta(\log r)=-0.26 \pm 0.15$; Schombert et al. 1993) although the spread of values is quite large. For nearby RGs, Govoni et al. (2000) also found slightly steeper colour gradient $(\langle\Delta(B-R) / \Delta \log r\rangle=-0.16 \pm 0.17)$ than that of normal ellipticals $(\langle\Delta(B-R) / \Delta \log r\rangle=-0.09 \pm 0.07$; Peletier et al. 1990).

In Fig. 8 we show the distribution of the $R-H$ colour gradient for the combined sample of BL Lacs. For the majority of the objects the colour gradient is confined between -1.2 to 0.4 (average $\Delta(R-H) / \Delta(\log r) \sim-0.3)$ while for normal ellipticals (Peletier et al. 1990; Schombert et al. 1993), there is a narrower spread. A similar larger spread for the $B-R$ colour gradient was found by Govoni et al. (2000) for low $z$ RGs.

Note that there are some exceptions with a slightly positive gradient, i.e. bluer colour toward the center, e.g. in Mrk 180, 1ES $1426+428$ and 1ES 1853+671. More intriguingly, we confirm the result of $\mathrm{C} 03$ of a significant tail in the distribution with very strong red gradients toward the center, e.g. in Mrk 501, BL Lac and PKS 2201+04. These colour differences could be either intrinsic or due to central dust extinction in the host galaxies. Significant amount of dust in the central regions of RGs is often detected from HST imaging (e.g. Verdoes Kleijn et al. 1999; Capetti et al. 2000) as dust lanes or dusty disks. These small scale features are in general, however, undetectable in ground-based observations. 

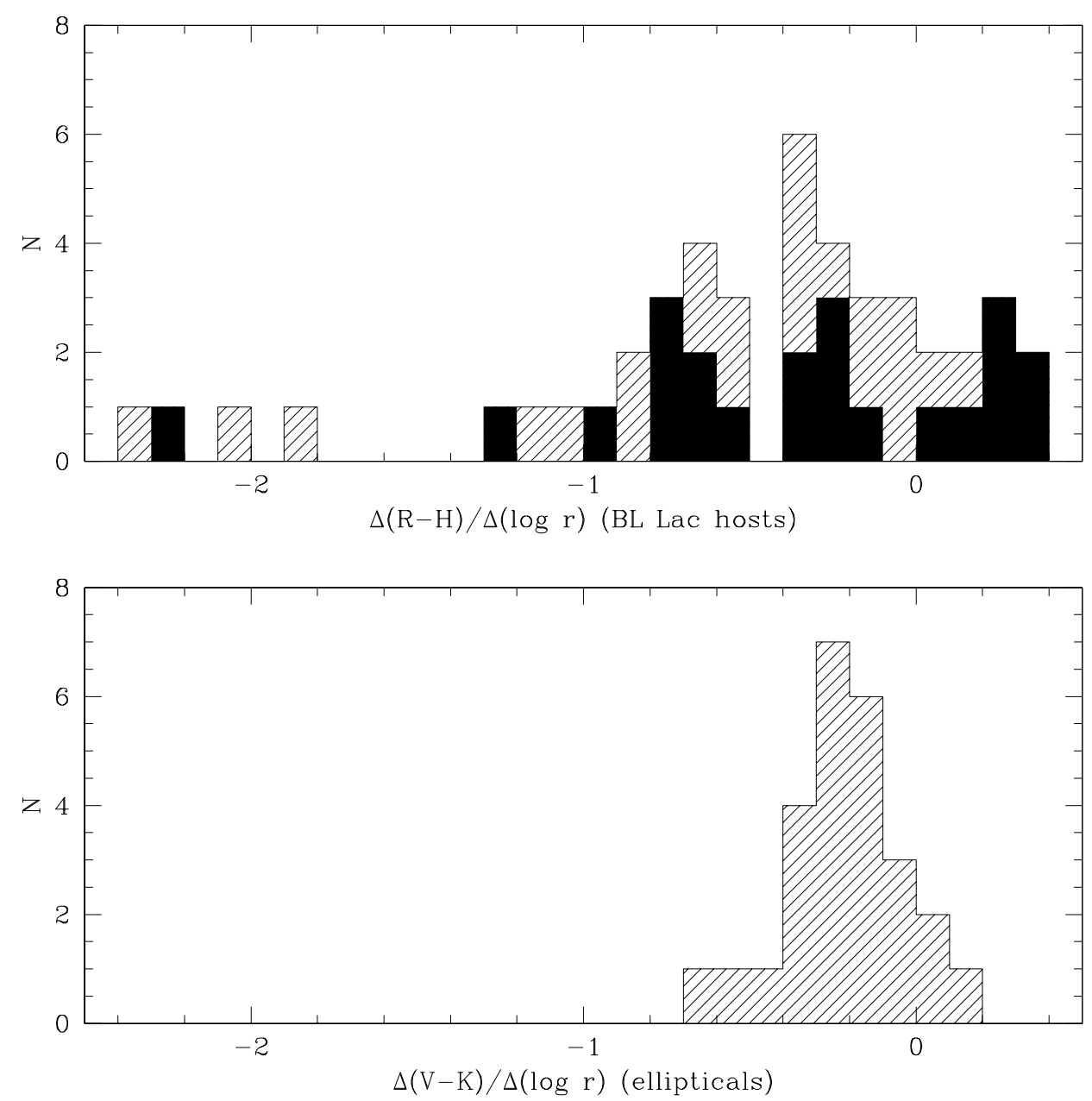

Fig. 8. Upper panel: histogram of the host galaxy $R-H$ colour gradient for all the BL Lac samples. The solid histogram shows the BL Lacs in this work. Lower panel: histogram of the host galaxy $V-K$ colour gradient for normal ellipticals (from Peletier et al. 1990; Schombert et al. 1993).

\section{Summary and conclusions}

We have presented homogeneous NIR imaging of a sample of 23 low redshift $(z \leq 0.3)$ BL Lac objects that were previously investigated in the optical band. All the new observed BL Lacs are clearly resolved, and their host galaxies well described in the NIR by an elliptical model. These new observations are combined with previous NIR data (K98, S00, C03) in order to study the NIR properties of the host galaxies in a sizeable sample (41 BL Lacs).

The global properties of the BL Lac host galaxies (average luminosity $M(H)=-25.8 \pm 0.7$ and bulge scale length $R(\mathrm{e})=$ $7.8 \pm 4.1 \mathrm{kpc}$ ) confirm previous suggestions that also in the NIR BL Lac hosts are luminous (massive) early type galaxies with structural and photometrical characteristics indistinguishable from those of low redshift RGs and inactive ellipticals. The NIR $r_{\mathrm{e}}-\mu_{\mathrm{e}}$ relation for BL Lac host galaxies is similar to that of normal ellipticals and RGs. Thus they appear to be also dynamically similar and occupy the same region of this projection of the fundamental plane as other ellipticals. This indicates that all massive elliptical galaxies can experience nuclear activity with little influence on their global structure.
There is increasing evidence that the photometric properties of elliptical galaxies (at least of those in nearby clusters) can be explained in terms of a single burst of SF at high redshift, followed by passive stellar evolution (e.g. Stanford et al. 1998, and references therein). On the other hand, on-going galaxy interactions and/or minor mergers, often invoked to explain and sustain the nuclear activity (e.g. Heckman 1990), could induce a recent episode of SF.

Our NIR study combined with optical data was able to address the issue of the optical-NIR colour and colour gradient of BL Lac host galaxies using a large and homogeneous dataset. The results confirm and extend previous findings (also reported for RGs and quasars) that the $R-H$ colour and colour gradient distributions of the BL Lac hosts are much wider than those for normal ellipticals with old stellar populations, and many BL Lacs have bluer hosts and/or steeper colour gradients than those observed in normal ellipticals. Assuming that the blue colours are caused by a young stellar population component, it may represent the link between the SF episode caused by an interaction/merging event and the onset of the nuclear activity. However, both the optical and NIR images of BL Lac hosts show few obvious signs of interaction (e.g. tidal tails, asymmetries, secondary nuclei). This may require a significant 
time delay (at least $100 \mathrm{Myr}$ ) between the event with associated SF episodes and the start of the nuclear activity.

Acknowledgements. Nordic Optical Telescope is operated on the island of La Palma jointly by Denmark, Finland, Iceland, Norway and Sweden, in the Spanish Observatorio del Roque de los Muchachos of the Instituto de Astrofisica de Canarias. This research has made use of the NASA/IPAC Extragalactic Database (NED), which is operated by the Jet Propulsion Laboratory, California Institute of Technology, under contract with the National Aeronautics and Space Administration. J.K.K. acknowledges financial support from the Academy of Finland, project 8201017.

\section{References}

Abraham, R. G., McHardy, I. M., \& Crawford, C. S. 1991, MNRAS, 252,482

Aragon-Salamanca, A., Baugh, C. M., \& Kauffmann, G. 1998, MNRAS, 297, 427

Barth, A. J., Ho, L. C., \& Sargent, W. L. W. 2003, ApJ, 583, 134

Bettoni, D., Falomo, R., Fasano, G., et al. 2001, A\&A, 380, 471

Blandford, R. D., \& Rees, M. J. 1978, Pittsburgh conference on BL Lac objects, ed. A. M. Wolfe, 328

Bower, R. G., Lucey, J. R., \& Ellis, R. S. 1992a, MNRAS, 254, 589

Bower, R. G., Lucey, J. R., \& Ellis, R. S. 1992b, MNRAS, 254, 607

Bressan, A., Granato, G. L., \& Silva, L. 1998, A\&A, 332, 135

Capaccioli, M., Caon, N., \& D’Onofrio, M. 1992, MNRAS, 259, 323

Capetti, A., de Ruiter, H. R., Fanti, R., et al. 2000, A\&A, 362, 871

Cardelli, J. A., Clayton, G. C., \& Mathis, J. S. 1989, ApJ, 345, 245

Cheung, C. C., Urry, C. M., Scarpa, R., \& Giavalisco, M. 2003, ApJ, $599,155(\mathrm{C} 03)$

de Vries, W. H., O’Dea, C. P., Baum, S. A., et al. 1998, ApJ, 503, 156

Djorgovski, S., \& Davis, M. 1987, ApJ, 313, 59

Falomo, R. 1996, MNRAS, 283, 241

Falomo, R., \& Kotilainen, J. K. 1999, A\&A, 352, 85

Falomo, R., Kotilainen, J., Pursimo, T., et al. 1997a, A\&A, 321, 374

Falomo, R., Urry, C. M., Pesce, J. E., et al. 1997b, ApJ, 476, 113

Falomo, R., Scarpa, R., Treves, A., \& Urry, C. M. 2000, ApJ, 542, 731

Falomo, R., Kotilainen, J. K., \& Treves, A. 2002, ApJ, 569, L35

Falomo, R., Kotilainen, J. K., Carangelo, N., \& Treves, A. 2003, ApJ, 595,624

Govoni, F., Falomo, R., Fasano, G., \& Scarpa, R. 2000, A\&A, 353, 507

Heckman, T. M. 1990, in Paired and interacting galaxies, ed. J. W. Sulentic, W. C. Keel, \& C. M. Telesco, NASA Conf. Publ., 3098, 359

Heidt, J., Nilsson, K., Sillanpää, A., Takalo, L. O., \& Pursimo, T. 1999, A\&A, 341, 683
Hunt, L. K., Mannucci, F., Testi, L., et al. 1998, AJ, 115, 2594

Jahnke, K., Kuhlbrodt, B., \& Wisotzki, L. 2003, MNRAS, in press [arXiv: astro-ph/0311123]

Jannuzi, B. T., Yanny, B., \& Impey, C. 1997, ApJ, 491, 146

Kodama, T., \& Arimoto, N. 1997, A\&A, 320, 41

Kormendy, J. 1977, ApJ, 218, 333

Kormendy, J., \& Djorgovski, S. 1989, ARA\&A, 27, 235

Kotilainen, J. K., Falomo, R., \& Scarpa, R. 1998, A\&A, 336, 479 (K98)

McCarthy, P. 1993, PASP, 105, 1051

Mobasher, B., Sharples, R. M., \& Ellis, R. S. 1993, MNRAS, 263, 560

Neugebauer, G., Matthews, K., Soifer, B. T., \& Elias, J. H. 1985, ApJ, 298, 275

Nilsson, K., Pursimo, T., Heidt, J., et al. 2003, A\&A, 400, 95

Pahre, M. A., Djorgovski, S. G., \& de Carvalho, R. R. 1995, ApJ, 453, L17

Peletier, R. F., Valentijn, E. A., \& Jameson, R. F. 1990, A\&A, 233, 62

Pursimo, T., Nilsson, K., Takalo, L. O., et al. 2002, A\&A, 381, 810

Recillas-Cruz, E., Carrasco, L., Serrano, A., \& Cruz-Gonzalez, I. 1990, A\&A, 229, 64

Scarpa, R., \& Falomo, R. 1997, A\&A, 325, 109

Scarpa, R., Urry, C. M., Falomo, R., Pesce, J. E., \& Treves, A. 2000a, ApJ, 532, 740

Scarpa, R., Urry, C. M., Padovani, P., Calzetti, D., \& O'Dowd, M. 2000b, ApJ, 544, 258 (S00)

Schade, D., Boyle, B. J., \& Letawsky, M. 2000, MNRAS, 315, 498

Schombert, J. M., Hanlan, P. C., Barsony, M., \& Rakos, K. D. 1993, AJ, 106, 923

Stanford, S. A., Eisenhardt, P. R., \& Dickinson, M. 1998, ApJ, 492, 461

Stickel, M., Fried, J. W., \& Kühr, H. 1993, A\&AS, 98, 393

Stocke, J. T., Wurtz, R. E., \& Perlman, E. S. 1995, ApJ, 454, 55

Tamura, N., Kobayashi, C., Arimoto, N., Kodama, T., \& Ohta, K. 2000, AJ, 119, 2134

Taylor, G. L., Dunlop, J. S., Hughes, D. H., \& Robson, E. I. 1996, MNRAS, 283, 930

Thuan, T. X., \& Puschell, J. J. 1989, ApJ, 346, 34

Urry, C. M., \& Padovani, P. 1995, PASP, 107, 803

Urry, C. M., Scarpa, R., O’Dowd, M., et al. 2000, ApJ, 532, 816

van der Marel, R. 1999, AJ, 117, 744

Verdoes Kleijn, G. A., Baum, S. A., de Zeeuw, P. T., \& O'Dea, C. P. 1999, AJ, 118, 2592

Willott, C. J., Rawlings, S., Jarvis, M. J., \& Blundell, K. M. 2003, MNRAS, 339, 173

Wise, M. W., \& Silva, D. R. 1996, ApJ, 461, 155

Wright, S. C., McHardy, I. M., Abraham, R. G., \& Crawford, C. S. 1998, MNRAS, 296, 961

Wurtz, R., Stocke, J. T., \& Yee, H. K. C. 1996, ApJS, 103, 109 


\section{Online Material}


J. K. Kotilainen and R. Falomo: NIR imaging of BL Lac hosts, Online Material $p 2$

Table 1. The sample and journal of observations ${ }^{a}$.

\begin{tabular}{|c|c|c|c|c|c|c|c|}
\hline Name & $\begin{array}{l}L / H \\
\text { (2) }\end{array}$ & (3) & $\begin{array}{l}V \\
(4)\end{array}$ & $\begin{array}{l}M(B) \\
(5)\end{array}$ & $\begin{array}{l}\text { Date } \\
(6)\end{array}$ & $\begin{array}{l}T(\exp ) \\
\min \\
(7)\end{array}$ & $\begin{array}{l}F W H M \\
\operatorname{arcsec} \\
\text { (8) }\end{array}$ \\
\hline $1 \mathrm{ES} 0120+340$ & $\mathrm{H}$ & 0.272 & 15.2 & -26.0 & $20 / 07 / 2002$ & 32 & 0.75 \\
\hline MS 0158.5+0019 & $\mathrm{H}$ & 0.298 & 18.0 & -23.4 & $17-18 / 07 / 2002$ & 44 & 0.85 \\
\hline MS 0205.7+3509 & $\mathrm{H}$ & $0.318:$ & 19.2 & -22.3 & $20 / 07 / 2002$ & 48 & 0.65 \\
\hline MS $0317.0+1834$ & $\mathrm{H}$ & 0.190 & 18.1 & -22.2 & $18 / 07 / 2002$ & 32 & 0.80 \\
\hline Mrk 180 & $\mathrm{H}$ & 0.045 & 14.5 & -22.1 & $17 / 07 / 2002$ & 20 & 0.60 \\
\hline 1ES $1218+304$ & $\mathrm{H}$ & 0.182 & 16.5 & -23.0 & $18 / 07 / 2002$ & 32 & 0.70 \\
\hline MS $1221.8+2452$ & $\mathrm{H}$ & $0.218:$ & 17.4 & -22.7 & $19 / 07 / 2002$ & 32 & 0.85 \\
\hline MS $1229.2+6430$ & $\mathrm{H}$ & 0.164 & 16.9 & -23.1 & $17 / 07 / 2002$ & 40 & 0.60 \\
\hline 1ES $1255+244$ & $\mathrm{H}$ & 0.140 & 15.4 & -24.3 & 20/07/2002 & 27 & 0.70 \\
\hline 1ES $1426+428$ & $\mathrm{H}$ & 0.129 & 16.5 & -22.5 & $17 / 07 / 2002$ & 30 & 0.70 \\
\hline 1ES $1440+122$ & $\mathrm{H}$ & 0.162 & 17.1 & -22.9 & $18 / 07 / 2002$ & 32 & 0.90 \\
\hline MS $1458.8+2249$ & $\mathrm{H}$ & 0.235 & 16.8 & -24.0 & $20 / 07 / 2002$ & 32 & 0.60 \\
\hline MS $1552.1+2020$ & $\mathrm{H}$ & 0.273 & 17.7 & -23.0 & $18 / 07 / 2002$ & 40 & 0.80 \\
\hline Mrk 501 & $\mathrm{H}$ & 0.034 & 13.8 & -22.4 & $17 / 07 / 2002$ & 10 & 0.70 \\
\hline I Zw 187 & $\mathrm{H}$ & 0.055 & 16.0 & -21.1 & $17 / 07 / 2002$ & 30 & 0.60 \\
\hline 3C 371 & $\mathrm{~L}$ & 0.051 & 14.2 & -22.7 & $18 / 07 / 2002$ & 23 & 0.75 \\
\hline 1ES $1853+671$ & $\mathrm{H}$ & 0.212 & 16.4 & -24.2 & $17 / 07 / 2002$ & 30 & 0.85 \\
\hline 1ES $1959+650$ & $\mathrm{H}$ & 0.048: & 12.8 & -24.5 & $20 / 07 / 2002$ & 16 & 0.55 \\
\hline BL Lac & $\mathrm{L}$ & 0.069 & 14.7 & -22.4 & $17 / 07 / 2002$ & 28 & 0.65 \\
\hline PKS 2201+04 & $\mathrm{L}$ & 0.027 & 15.2 & & $19 / 07 / 2002$ & 32 & 0.65 \\
\hline PKS 2254+074 & $\mathrm{L}$ & 0.194 & 16.4 & -23.3 & $19 / 07 / 2002$ & 32 & 0.70 \\
\hline 1ES $2326+174$ & $\mathrm{H}$ & 0.213 & 16.8 & -23.8 & $17 / 07 / 2002$ & 40 & 0.70 \\
\hline 1ES $2344+514$ & $\mathrm{H}$ & 0.044 & 15.5 & -21.6 & $18 / 07 / 2002$ & 24 & 0.75 \\
\hline
\end{tabular}

${ }^{a}$ Column (1) gives the name of the object; (2) high-frequency (H) or low-frequency (L) peaked object; (3) the redshift; (4) $V$-band apparent magnitude; (5) B-band absolute magnitude; (6) the date of observation; (7) total exposure time; and (8) seeing FWHM. 
J. K. Kotilainen and R. Falomo: NIR imaging of BL Lac hosts, Online Material p 3
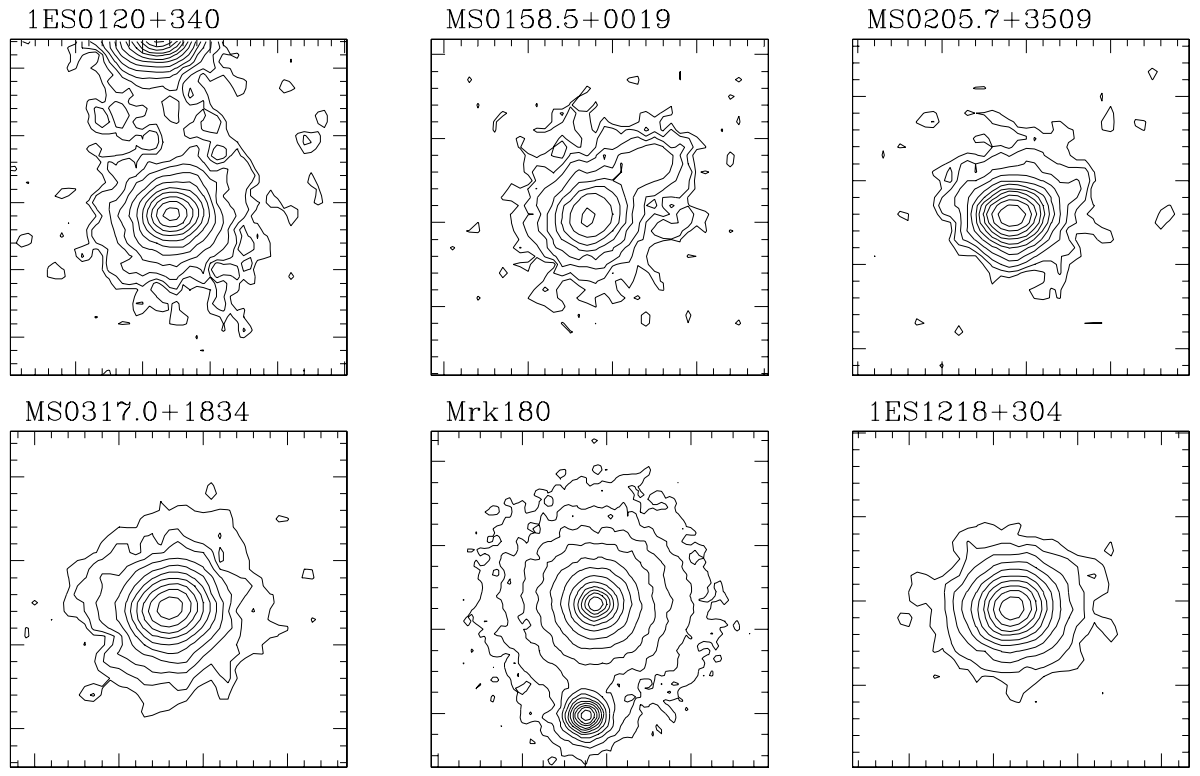

MS1221.8+2452

MS1229.2+6430
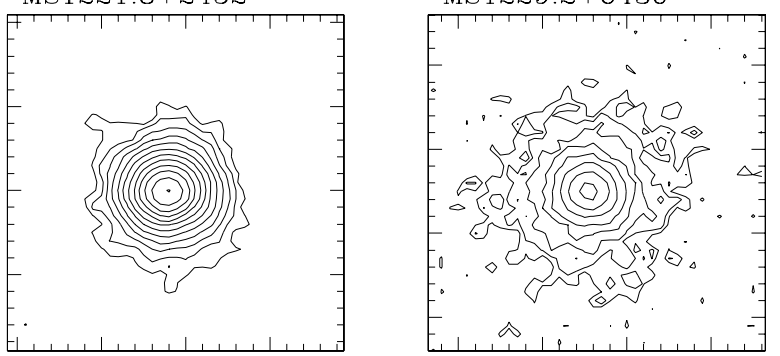

$1 \mathrm{ES} 1255+244$
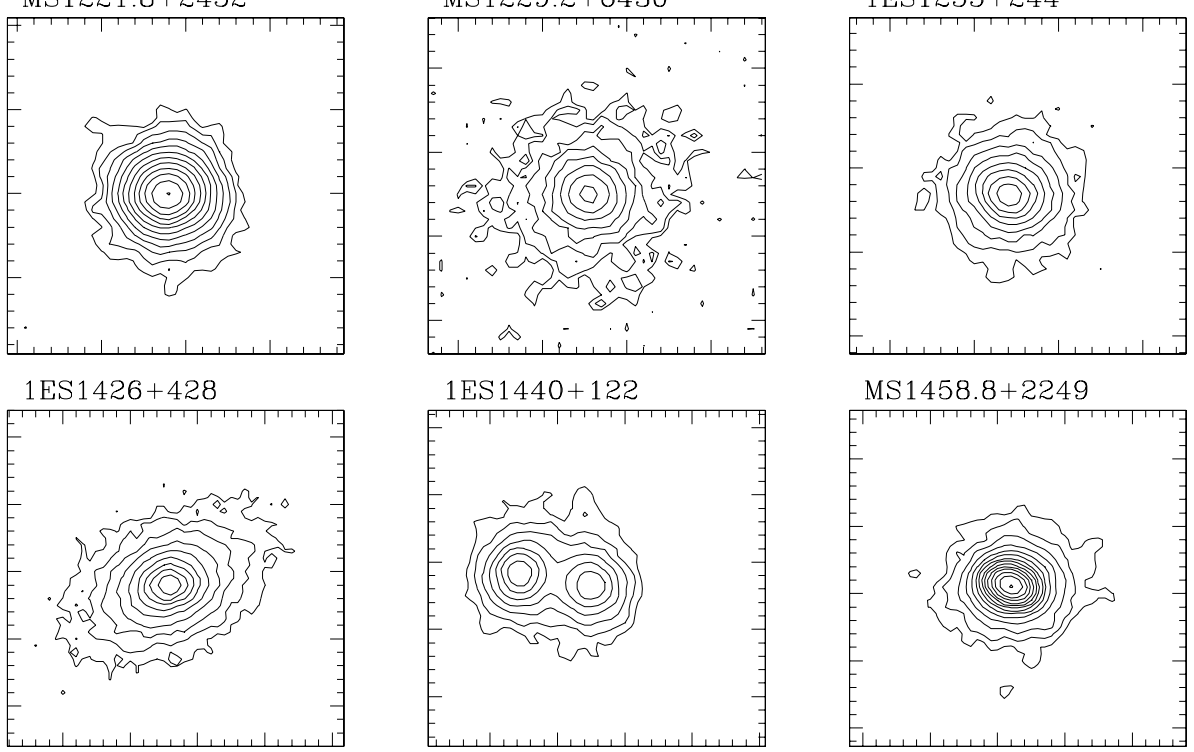

MS1458.8+2249

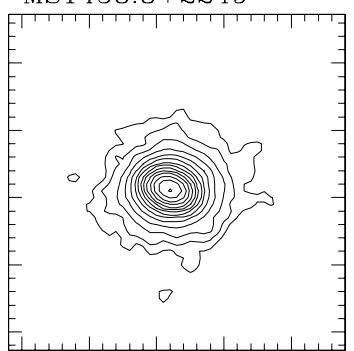

Fig. 1. Contour images of the BL Lac objects in the $H$-band. The distance between major tick marks is 10 px (2'. 35$)$. Successive isophotes are separated by $0.5 \mathrm{mag}$ intervals. North is up and east to the left. The host galaxy is resolved in all objects. 
J. K. Kotilainen and R. Falomo: NIR imaging of BL Lac hosts, Online Material p 4
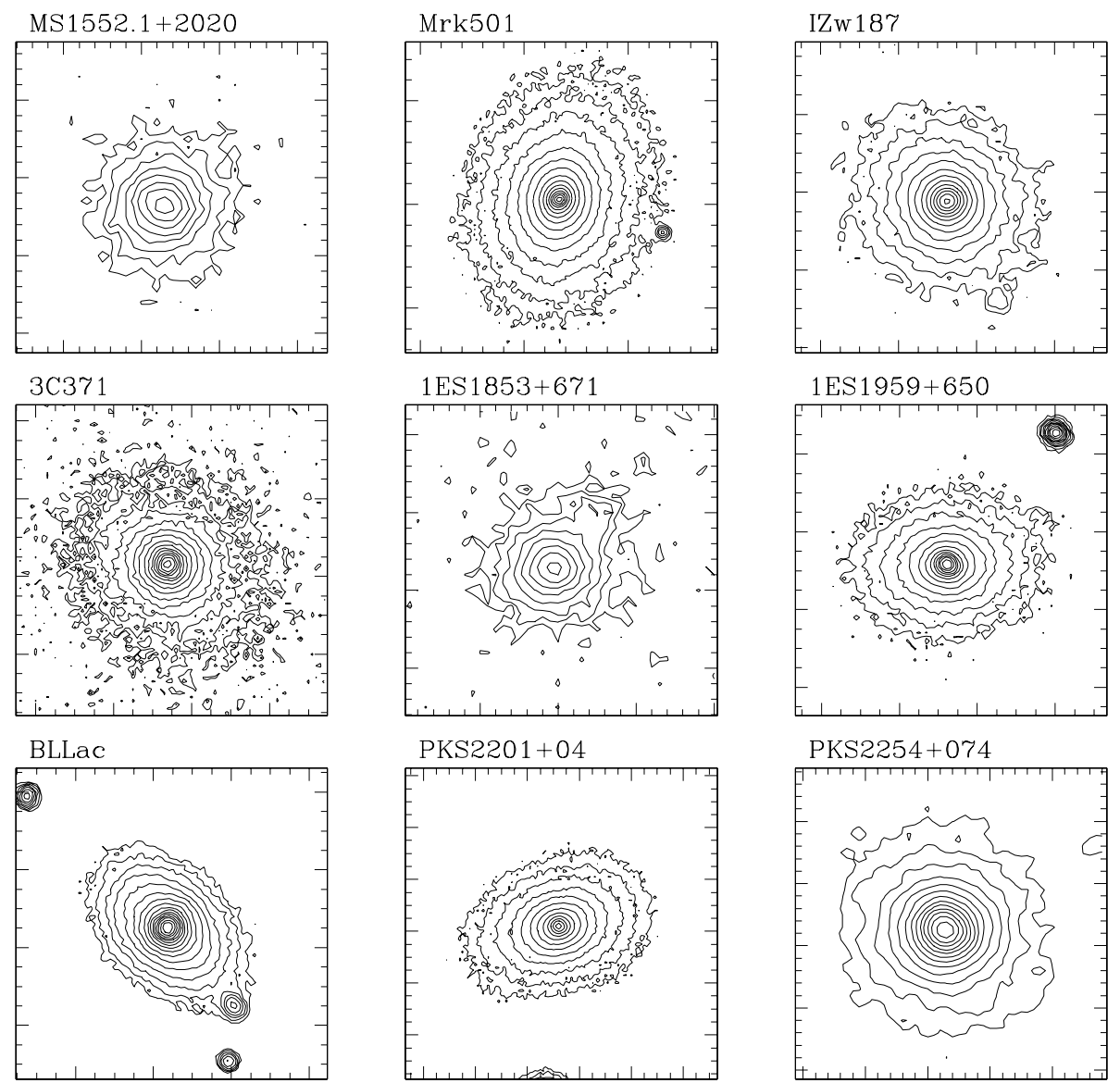

$1 \mathrm{ES} 2326+174$

1ES2344+514
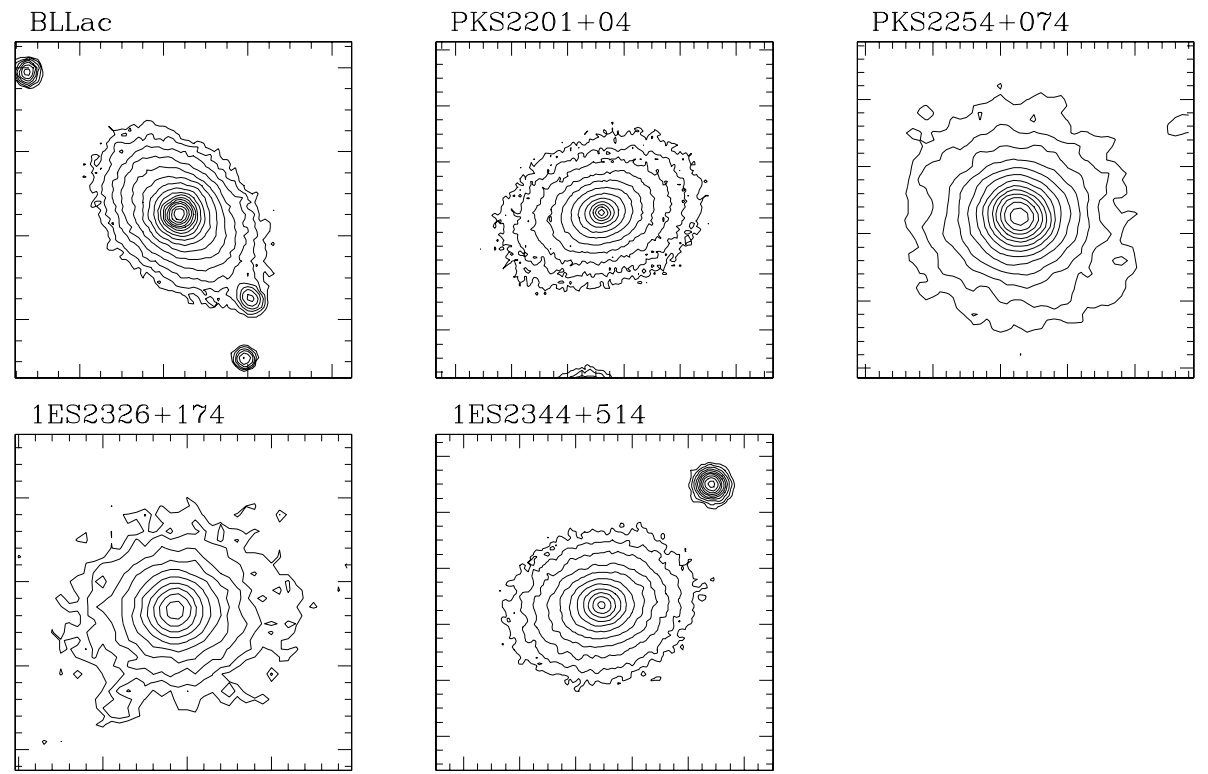

Fig. 1. continued. 